\title{
Novel Virulent Bacteriophage $\Phi$ SG005, Which Infects Streptococcus gordonii, Forms a Distinct Clade among Streptococcus Viruses
}

\author{
Jumpei Fujiki ${ }^{1} @$, Shin-ichi Yoshida ${ }^{1}$, Tomohiro Nakamura ${ }^{1}$, Keisuke Nakamura ${ }^{1}$, Yurika Amano ${ }^{1}$, \\ Keita Nishida ${ }^{1}$, Keitaro Nishi ${ }^{2}$, Michihito Sasaki ${ }^{3}{ }^{(1)}$, Tomohito Iwasaki ${ }^{4}$, Hirofumi Sawa ${ }^{3,5,6}{ }^{(1)}$, \\ Hitoshi Komatsuzawa ${ }^{7}$, Hiroshi Hijioka ${ }^{2}$ and Hidetomo Iwano ${ }^{1, *(\mathbb{D}}$
}

\section{check for} updates

Citation: Fujiki, J.; Yoshida, S.-i.; Nakamura, T.; Nakamura, K.; Amano, Y.; Nishida, K.; Nishi, K.; Sasaki, M.; Iwasaki, T.; Sawa, H.; et al. Novel Virulent Bacteriophage $\Phi$ SG005, Which Infects Streptococcus gordonii, Forms a Distinct Clade among Streptococcus Viruses. Viruses 2021, 13, 1964. https://doi.org/10.3390/ v13101964

Academic Editor: Terje Dokland

Received: 7 August 2021

Accepted: 25 September 2021

Published: 29 September 2021

Publisher's Note: MDPI stays neutral with regard to jurisdictional claims in published maps and institutional affiliations.

Copyright: (C) 2021 by the authors. Licensee MDPI, Basel, Switzerland. This article is an open access article distributed under the terms and conditions of the Creative Commons Attribution (CC BY) license (https:/ / creativecommons.org/licenses/by/ $4.0 /)$.
1 Laboratory of Veterinary Biochemistry, Department of Veterinary Medicine, Rakuno Gakuen University, Ebetsu 069-8501, Japan; j-fujiki@rakuno.ac.jp (J.F.); Shinichi.Yoshida@kaneka.co.jp (S.-i.Y.); s21941005@stu.rakuno.ac.jp (T.N.); s21661158@stu.rakuno.ac.jp (K.N.); s21661157@stu.rakuno.ac.jp (Y.A.); nk.0418trbr@gmail.com (K.N.)

2 Department of Maxillofacial Diagnostic and Surgical Science, Field of Oral and Maxillofacial Rehabilitation, Graduate School of Medical and Dental Sciences, Kagoshima University, Kagoshima 890-8544, Japan; nsktr.248@gmail.com (K.N.); zio@dent.kagoshima-u.ac.jp (H.H.)

3 Division of Molecular Pathobiology, International Institute for Zoonosis Control, Hokkaido University, Sapporo 001-0020, Japan; m-sasaki@czc.hokudai.ac.jp (M.S.); h-sawa@czc.hokudai.ac.jp (H.S.)

4 Department of Food Science and Human Wellness, College of Agriculture, Food and Environment Science, Rakuno Gakuen University, Ebetsu 069-8501, Japan; iwasaki@rakuno.ac.jp

5 International Collaboration Unit, International Institute for Zoonosis Control, Hokkaido University, Sapporo 001-0020, Japan

6 One Health Research Center, Hokkaido University, Sapporo 001-0020, Japan

7 Department of Bacteriology, Graduate School of Biomedical and Health Sciences, Hiroshima University, Hiroshima 734-8553, Japan; komatsuz@hiroshima-u.ac.jp

* Correspondence: h-iwano@rakuno.ac.jp; Tel./Fax: +81-11-388-4885

Abstract: Bacteriophages are viruses that specifically infect bacteria and are classified as either virulent phages or temperate phages. Despite virulent phages being promising antimicrobial agents due to their bactericidal effects, the implementation of phage therapy depends on the availability of virulent phages against target bacteria. Notably, virulent phages of Streptococcus gordonii, which resides in the oral cavity and is an opportunistic pathogen that can cause periodontitis and endocarditis have previously never been found. We thus attempted to isolate virulent phages against $S$. gordonii. In the present study, we report for the first time a virulent bacteriophage against S. gordonii, ФSG005, discovered from drainage water. $\Phi S G 005$ is composed of a short, non-contractile tail and a long head, revealing Podoviridae characteristics via electron microscopic analysis. In turbidity reduction assays, ФSG005 showed efficient bactericidal effects on S. gordonii. Whole-genome sequencing showed that the virus has a DNA genome of 16,127 bp with 21 coding sequences. We identified no prophagerelated elements such as integrase in the $\Phi$ SG005 genome, demonstrating that the virus is a virulent phage. Phylogenetic analysis indicated that $\Phi$ SG005 forms a distinct clade among the streptococcus viruses and is positioned next to streptococcus virus $\mathrm{C} 1$. Molecular characterization revealed the presence of an anti-CRISPR (Acr) IIA5-like protein in the $\Phi$ SG005 genome. These findings facilitate our understanding of streptococcus viruses and advance the development of phage therapy against S. gordonii infection.

Keywords: bacteriophage; streptococcus virus; phage therapy; Streptococcus gordonii

\section{Introduction}

Bacteriophages, which are viruses that infect bacterial cells, are ubiquitous in nature. Phage lifecycles are divided into two major categories, lysogenic and lytic [1,2]. In the lysogenic cycle, temperate phages infect host bacterial cells but are usually integrated into 
the host genome at attachment sites such as att $P$, att $R$, and attL by phage-encoded integrase, which does not lead to bacterial lysis [3]. This integrated form of phage is called a prophage. Conversely, in the lytic cycle, phages never form prophages and can kill host bacterial cells efficiently. Thus, virulent phages, which proliferate remarkably through the lytic cycle, have received significant attention as possible therapeutic agents against bacterial infection. Notably, phages are promising as an anti-microbial resistance (AMR) control option [2,4-6], and phages exhibit specific infectivity against bacteria, which allows for phage therapy against bacterial infection without the disruption of associated normal microbiota [7-9]. However, whether we can implement phage therapy efficiently depends on the availability of virulent phage. Phage therapy towards some kinds of bacteria has not been developed because virulent phages with efficient bactericidal effects against these bacteria have not been found [10-12].

Phage infections are closely associated with host genome diversification. Phages are the most abundant organisms in the biosphere, and prophage sequences have been detected in almost all sequenced bacterial genomes, which indicates that phages drive bacterial evolution via gene transfer and phage resistance. The CRISPR/Cas9 system, which is a gene editing technology platform, was first discovered in Streptococcus pyogenes as an adaptive immune response against phage infection [13]. In this system, bacteria can "memorize" exogenous nucleotide sequences and integrate them into the CRISPR array in the form of CRISPR spacers [14]. In addition, prophages or prophage remnants are frequently found in various streptococci genomes [15-17]. This viral information derived from the sequenced hosts contributes to detailed understanding of streptococcus viruses; however, there is limited information about virulent phages that infect streptococci compared to other virulent phages such as Escherichia viruses and Pseudomonas viruses. Notably, virulent phages against Group B and some viridans streptococci such as S. agalactiae and S. gordonii have not been reported previously.

S. gordonii is a gram-positive facultative anaerobe that inhabits the oral cavities of several animal species, including humans [18]. Although S. gordonii is categorized as an opportunistic pathogen, it has been reported that this bacterium elicits periodontitis through an initial colonization of the tooth surface, which promotes the aggregation of other oral bacteria, such as Porphyromonas gingivalis, leading to pathogenic conditions $[19,20]$. In addition, oral bleeding allows S. gordonii to enter into blood circulation, providing it with the opportunity to reach various organs $[18,21]$. Previous microbiome analysis has detected S. gordonii colonization in the heart valves of infective endocarditis patients [22]. S. gordonii is thus a candidate target of the oral microbiota for the control of inflammation.

In the present study, we discovered for the first time a novel virulent phage against S. gordonii, $\Phi$ SG005, and characterized its molecular features. These findings facilitate our understanding of streptococcus viruses and provide insight into the development of phage therapy against $S$. gordonii.

\section{Materials and Methods}

\subsection{Bacterial Strains}

S. gordonii ATCC10558 [23] was grown at $37^{\circ} \mathrm{C}$ anaerobically with AnaeroPack (Mitsubishi Gas Chemical, Tokyo, Japan) in Gifu anaerobic medium (GAM) broth (Nissui, Tokyo, Japan) and used for downstream assays as described below.

\subsection{Bacteriophage Isolation}

Phages were isolated by the double-layer method as described elsewhere [4]. In brief, drainage water collected from Kagoshima University School of Dentistry (approved by the Committee on Ethics of Kagoshima University: permit number 180294) was centrifuged at $10,000 \times g$ for $15 \mathrm{~min}$. The resultant supernatants were filtered through $0.45-\mu \mathrm{m}$ syringe filters (Advantec, Tokyo, Japan) and subject to polyethylene glycol $6000-\mathrm{NaCl}$ centrifugation. This concentrated sample was mixed with S. gordonii ATCC10558 and overlaid on brain heart infusion (BHI) agar (Becton Dickinson, Franklin Lakes, NJ, USA) plates with 
GAM top agar containing $0.5 \%$ agarose ME (Iwai Chemicals, Tokyo, Japan) and incubated at $37^{\circ} \mathrm{C}$ anaerobically overnight. Single plaques were collected and purified by repeatedly plating and picking four times.

\subsection{Bacteriophage Preparation}

For downstream assays, an isolated phage was amplified by the double-layer method $[4,5]$. In brief, S. gordonii ATCC10558 in GAM broth was mixed with phage and added to $3 \mathrm{~mL}$ of $0.5 \%$ GAM top agar. Subsequently, the mixture was overlaid on a BHI agar plate. After overnight incubation of the plate at $37^{\circ} \mathrm{C}$ anaerobically, $3 \mathrm{~mL}$ of SM buffer $(10 \mathrm{mM} \mathrm{MgSO}$, $100 \mathrm{mM} \mathrm{NaCl}, 0.01 \%$ gelatin, and $50 \mathrm{mM}$ Tris- $\mathrm{HCl}$ [pH 7.5]) was added to the plate, and the plate was incubated at room temperature for 1 to $2 \mathrm{~h}$ with shaking. The overlaid top agar was scraped off and homogenized with SM buffer by a glass spreader. The collected homogenate was purified using an Amicon Ultra membrane filter (Merck, Darmstadt, Germany) based on the phage on tap (PoT) method described by Bonilla et al. [24]. The viral titer of the phage was calculated as the number of plaques in a plaque assay using S. gordonii ATCC10558 as the host in accordance with previous reports [4] and is represented as plaque-forming units per milliliter $(\mathrm{pfu} / \mathrm{mL})$.

\subsection{Transmission Electron Microscopic Imaging}

Electron microscopic imaging was performed as described previously [5]. Purified phage samples were loaded onto copper grids (EMJapan, Tokyo, Japan). The grids were washed with SM buffer twice and stained with $2 \%$ uranyl acetate. Stained samples were observed with a Hitachi HT7700 transmission electron microscope (Hitachi, Ltd., Tokyo, Japan) at $75 \mathrm{kV}$.

\subsection{Whole-Genome Sequencing}

Purified phage samples were treated with DNase by using a TURBO DNase free kit (Thermo Fisher Scientific, San Jose, CA, USA) following the manufacturer's protocol. Then, the phage genome was extracted with the Phage DNA isolation kit (Novagen, Nottingham, UK). Libraries were prepared using a Nextere XT DNA library Preparation Kit (Illumina, San Diego, CA, USA). The whole genome of isolated phage was then 300-bp paired-end sequenced on a MiSeq platform (Illumina, San Diego, CA, USA) according to the manufacturer's instruction. The resulting 324,906 reads were trimmed of adaptors and low-quality bases (end and beginning of reads: $Q$ score $<20$, others: $Q$ score $<15$ ), and short reads $(<80 \mathrm{bp}$ ) were removed using Trimmomatic v0.39, resulting in a total of 300,169 reads (5584 $\times$ coverage). The generated sequence reads were assembled by de novo assembly using Unicycler v0.4.8. beta. Finally, the assembled sequences were annotated using PATRIC (https:/ / www.patricbrc.org (accessed on 10 September 2021)) with standard settings. The obtained genomic DNA sequence was submitted to the DDBJ/EMBL/GenBank databases under accession number LC628082 as summarized in Table 1.

Table 1. General genomic information of $\Phi S G 005$ and S. gordonii ATCC10558. The reference genome of S. gordonii ATCC10558 was obtained from the DDBJ/EMBL/GenBank databases.

\begin{tabular}{cccccc}
\hline Strain & Component & Length (bp) & G+C (\%) & CDS & Accession No. \\
\hline ФSG005 & Viral genome & 16,127 & 34.5 & 21 & LC_628082 \\
ATCC10558 & Chromosome & $2,187,611$ & 49.52 & 2039 & NZ_LS483341.1 \\
\hline
\end{tabular}

\subsection{Adsorption Assay}

Adsorption rates of the phage towards host strains were determined as described in a previous report [5]. Phage in SM buffer $\left(1.0 \times 10^{8} \mathrm{pfu} / \mathrm{mL}\right)$ was suspended 1:1 with S. gordonii ATCC10558 $\left(1.0 \times 10^{9} \mathrm{cfu} / \mathrm{mL}\right)$ at a multiplicity of infection (MOI) of 0.1 . After $0.5,1,5$, or $10 \mathrm{~min}$ incubation at room temperature, the samples were immediately centrifuged at $10,000 \times g$ for $10 \mathrm{~min}$, and the resultant supernatant containing unabsorbed phages was used for the plaque assay described above. 


\subsection{Co-Culture of the Phage and Host Strain}

The lytic activity of the phage against $S$. gordonii ATCC10558 was evaluated by turbidity reduction assays as previously reported $[4,6]$. In brief, phage was inoculated to S. gordonii ATCC10558 in mid-exponential phase at an MOI of 10,1, and 0.1, and the mixture was subsequently incubated at $37^{\circ} \mathrm{C}$ for $24 \mathrm{~h}$. The density of the culture was monitored using a plate reader (Sunrise Rainbow Thermos RC, TECAN, Grödig Austria) at $\mathrm{OD}_{590}$ every $1 \mathrm{~h}$. After $24 \mathrm{~h}$ incubation, the culture was harvested and washed with PBS three times. Thereafter, obtained samples were further incubated at $37^{\circ} \mathrm{C}$ anaerobically for $24 \mathrm{~h}$ on BHI agar to calculate viable cells as the number of colonies represented as colony-forming units per milliliter $(\mathrm{cfu} / \mathrm{mL})$. In addition, in accordance with previous reports [4,25], $3 \mu \mathrm{L}$ of diluted phage samples $\left(1.0 \times 10^{9}\right.$ to $\left.1.0 \times 10^{1} \mathrm{pfu} / \mathrm{mL}\right)$ in SM buffer was dropped onto a BHI agar plate overlaid with S. gordonii ATCC10558 to observe the lytic activity of phages by plaque formation.

\subsection{Bioinformatics Analysis}

A phylogenetic tree of phage whole-genomes was constructed by VICTOR [26] using the obtained viral nucleotide sequence. Amino acid sequences of DNA polymerase (CDS11), tail fiber (CDS15), CHAP-domain containing lysin (CDS12), and phage lysin (CDS13) were aligned with representative sequences of other known phages available in the National Center for Biotechnology Information (NCBI) database, and phylogenetic trees were constructed by MEGA X [27]. Prophage sequences within the genome of $S$. gordonii ATCC10558 were annotated and identified by Phage Search Tool Enhanced Release (PHASTER, https:/ / phaster.ca (accessed on 10 September 2021)). Annotation analysis was performed by PATRIC (https:/ / www.patricbrc.org (accessed on 10 September 2021)). The intergenomic similarity between phages was calculated by VIRIDIC [28].

\subsection{Statistical Analysis}

Statistical analysis was calculated by Tukey's test based on one-way ANOVA analysis from four independent experiments, and significance was set at $p<0.01$.

\section{Results}

\subsection{Isolation of Streptococcus Virus $\Phi S G 005$}

To isolate virulent phages against $S$. gordonii, we collected drainage water from the Kagoshima University School of Dentistry and purified it for phage isolation, because $S$. gordonii mainly inhabits the oral cavity [18]. The double-layer method using S. gordonii ATCC10558 as a host resulted in clear plaques as shown in Figure 1A, and subsequently one plaque was picked for isolation. The purified clone was subjected to morphological analysis. Electron microscopic imaging identified phage particles revealing a short, non-contractile tail and a relatively long polyhedral head (Figure 1B), which was classified morphologically as belonging to the Podoviridae family according to the report by Ackerman [29]. Long tail fibers were also observed in the electron micrograph (Figure 1B, lower panel), and this isolate was designated as $\Phi$ SG005. 

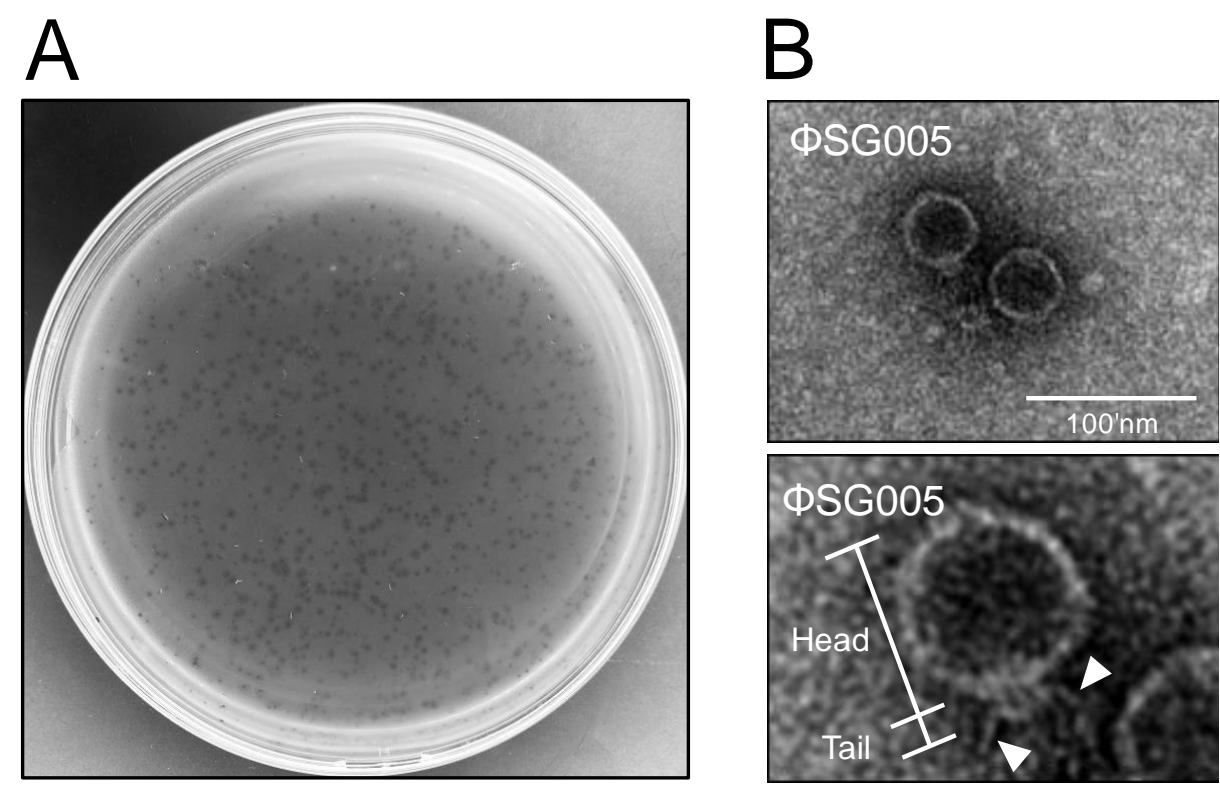

Figure 1. Isolation of a streptococcus virus, $\Phi S G 005$. (A) Plaques representing phages are observed on a lawn of S. gordonii ATCC10558. (B) Electron microscopic image showing phage particles exhibiting Podoviridae characteristics (upper panel). Lower panel indicates representative morphological structures of the viral particle including the head and tail. Arrowheads indicate the long tail fibers of the virus. Bar $=100 \mathrm{~nm}$.

\subsection{Lytic Activity of Streptococcus Virus $\Phi S G 005$}

We next assessed the lytic activity of $\Phi S G 005$. As shown in Figure 2A, more than 50\% of $\Phi$ SG005 adsorbed to host streptococcal strain ATCC10558 within 1 min, and about 90\% had adsorbed within 5 min. Co-culture of $\Phi S G 005$ and S. gordonii ATCC10558 demonstrated that $\Phi$ SG005 (MOI of 10 and 1) lysed the host strain efficiently from 3-5 h post-infection (hpi) and continued to suppress the growth of S. gordonii ATCC10558 at $24 \mathrm{hpi}$ (Figure 2B). The viable cells in the culture inoculated with $\Phi$ SG005 (MOI of 1 and 0.1 ) at 24 hpi decreased significantly compared with that of control (Mock). In addition, viable cells in the culture (MOI of 10) at 24 hpi were under the limit of detection (Figure 2C). Figure 2D clearly shows that SSG005 produced clear spots at high doses $\left(1.0 \times 10^{9}-1.0 \times 10^{6} \mathrm{pfu} / \mathrm{mL}\right)$ and plaques at low doses $\left(1.0 \times 10^{5}-1.0 \times 10^{3} \mathrm{pfu} / \mathrm{mL}\right)$, representing efficient bactericidal activity as "lysis from within".

\subsection{Genome Organization of $\Phi S G 005$}

Extracted DNA from $\Phi S G 005$ was whole-genome sequenced on a short-read platform. The obtained reads assembled through Unicycler resulted in a single linear contig with a total length of $16,127 \mathrm{bp}$, a G+C content of $34.5 \%$, and 21 coding sequences (CDS) as described in Table 1. The resultant full-length nucleotide sequence was subjected to a BLASTn search, which demonstrated that there are no similar viral sequences in the database, indicating that $\Phi S G 005$ possesses a previously uncharacterized genome. Predicted functions of each $\Phi$ SG005 CDS are annotated by PATRIC and PSI-BLAST analysis is summarized in Table 2, which shows that QSG005 possesses phage-associated genes such as an anti-CRISPR (Acr) IIA5-like protein (CDS2), phage capsid and scaffold (CDS5 and 6), phage tail fiber (CDS11), holin (CDS14), DNA polymerase (CDS15), and phage neck (CDS16). In addition, no phage-associated integrases were detected in the viral genome by gene annotation analysis, indicating that $\Phi S G 005$ is a virulent phage. In addition, the genome organization of $\Phi S G 005$ implied that CDS5-CDS12 encode viral structural proteins, while CDS13 and CDS14 encode the lysis module of QSG005 (Figure 3A). Notably, it was predicted that $\Phi S G 005$ possesses two lysin-related genes, a CHAP-domain containing lysin (CDS12) and phage endolysin (CDS13) by PATRIC; however, the $\Phi$ SG005 genome 
organization (Figure 3A) indicated that CDS12 is a tail-associated enzyme categorized as a structural component.

A

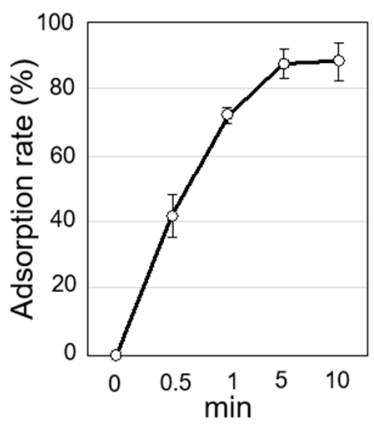

B

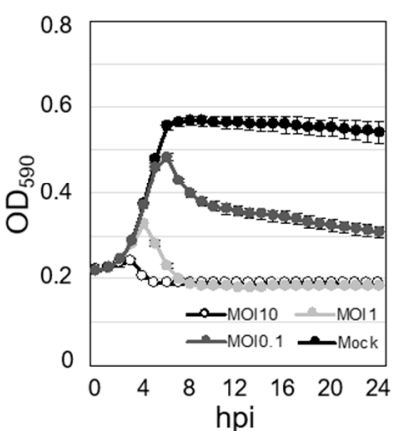

C

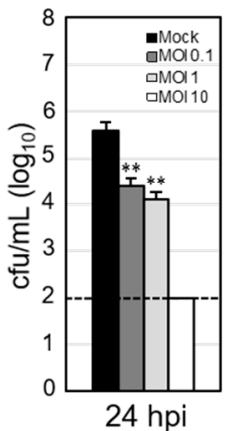

D

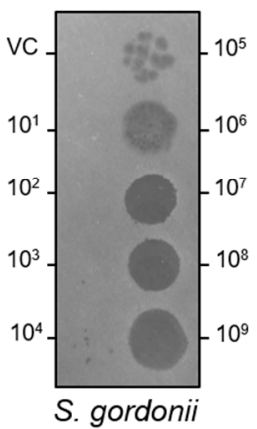

Figure 2. Lytic activity of $\Phi S G 005$ against S. gordonii ATCC10558. (A) Adsorption rate of $\Phi S G 005$ on S. gordonii ATCC10558 presented as means \pm SD $(n=4)$. (B) Lytic curves of S. gordonii ATCC10558 growing in the presence of $\Phi S G 005$ were obtained by monitoring the OD 590 until 24 hpi. $\Phi S G 005$ was inoculated at an MOI of 10,1, and 0.1. The individual points in each lytic curve are presented as means $\pm \mathrm{SD}(n=4)$. (C) Viable cells from the culture at 24 hpi are presented as means $\pm \mathrm{SD}(n=4) .1 .0 \times 10^{2} \mathrm{cfu} / \mathrm{mL}$ was the limit of detection. Significance was analyzed by Tukey's test based on one-way ANOVA analysis and is indicated by asterisks $\left({ }^{* *} p<0.01\right)$. (D) Representative spots and plaques on a lawn of S. gordonii ATCC10558 produced by $\Phi$ SG005. VC means vehicle control (SM buffer only).

Table 2. Products and predicted functions of proteins encoded by $\Phi$ SG005 CDSs.

\begin{tabular}{|c|c|c|c|c|}
\hline CDS & $\begin{array}{c}\text { Location } \\
\text { (Nucleotides) }\end{array}$ & Strand & Size (No. of Amino Acids) & Product or Predicted Function \\
\hline 1 & $1-237$ & + & 79 & hypothetical protein \\
\hline 2 & $239-670$ & + & 144 & anti-CRISPR protein AcrIIA5 \\
\hline 3 & $730-906$ & + & 59 & hypothetical protein \\
\hline 4 & $921-1079$ & + & 53 & Phage protein \\
\hline 5 & $1079-2239$ & + & 387 & Phage capsid and scaffold \\
\hline 6 & $2344-3306$ & + & 321 & Phage capsid and scaffold \\
\hline 7 & 3299-3955 & + & 219 & lower collar protein \\
\hline 8 & $3972-4841$ & + & 290 & hypothetical protein \\
\hline 9 & $4844-5569$ & + & 242 & hypothetical protein \\
\hline 10 & $5687-6234$ & + & 216 & hypothetical protein \\
\hline 11 & $6297-8021$ & + & 575 & Phage tail fiber \\
\hline 12 & $8023-9450$ & + & 476 & CHAP domain-containing phage lysin \\
\hline 13 & $10,375-9485$ & - & 297 & Phage endolysin \\
\hline 14 & $10,745-10,362$ & - & 128 & holin protein \\
\hline 15 & $13,088-10,797$ & - & 764 & DNA polymerase (EC 2.7.7.7), phage-associated \\
\hline 16 & $14,318-13,092$ & - & 409 & Phage neck \\
\hline 17 & $14,900-14,322$ & - & 193 & hypothetical protein \\
\hline 18 & $15,394-14,903$ & - & 164 & hypothetical protein \\
\hline 19 & $15,591-15,400$ & - & 64 & hypothetical protein \\
\hline 20 & $15,853-15,593$ & - & 87 & hypothetical protein \\
\hline 21 & $16,088-15,855$ & - & 78 & hypothetical protein \\
\hline
\end{tabular}




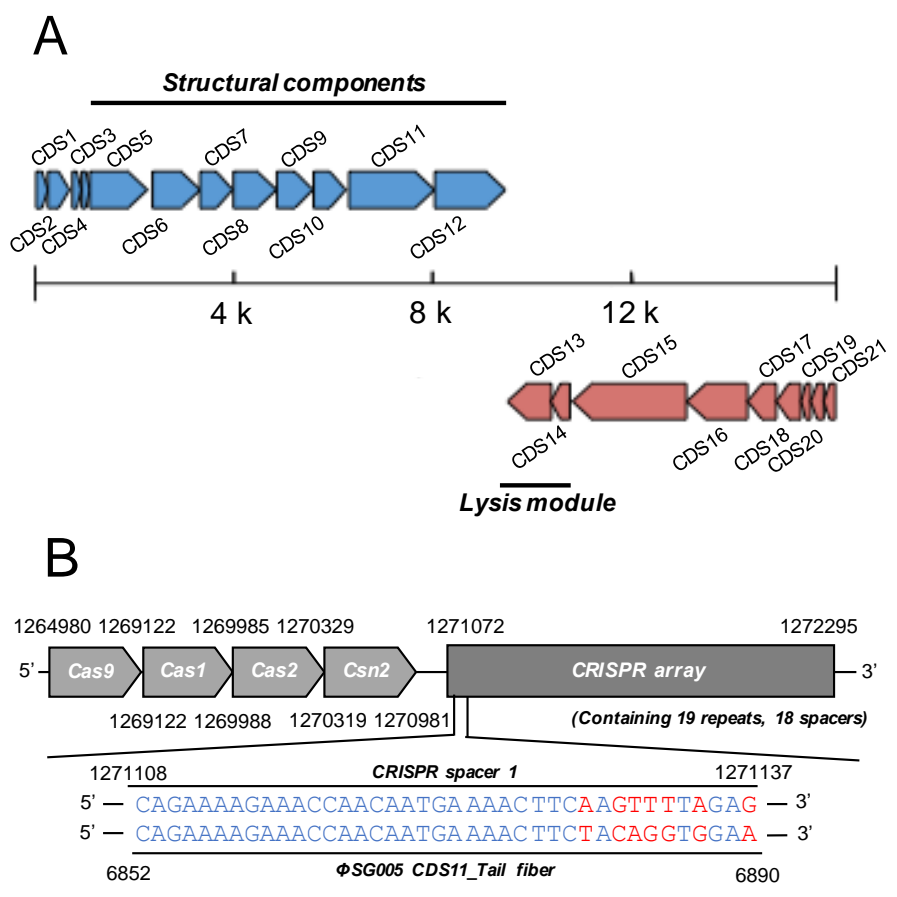

Figure 3. Genome structure of $\Phi S G 005$. (A) $\Phi S G 005$ possesses $16,127 \mathrm{bp}$ and a total of 21 CDSs indicated as blue or red arrows (complementary). Putative structural components (from CDS4 to CDS12) and putative lysis modules (CDS13 and CDS14) are marked by solid lines. (B) A section of the CRISPR-associated region in S. gordonii ATCC10558 is depicted. S. gordonii ATCC10558 harbors the type II CRISPR-Cas system composed of Cas9, Cas1, Cas2, and Csn2 (1,264,980-1,270,981 bp). The CRISPR array contains 19 repeats and 18 spacer sequences. One of the CRISPR spacers located at the most $5^{\prime}$ proximal region in the CRISPR array (represented as CRISPR spacer 1 ) shows high similarity with the sequence of the $\Phi S G 005$ CDS11 predicted tail fiber.

As the presence of Acr in the $\Phi S G 005$ was indicated, we then identified the CRISPRassociated sequences in S. gordonii ATCC10558 by PATRIC annotation, which revealed that S. gordonii ATCC10558 harbors a type II CRISPR-Cas system and a CRISPR array containing 19 repeats and 18 spacers (Figure 3B). The spacer-targeting analysis identified homologous sequences including a 7-nt mismatch at the $5^{\prime}$ region against the $\Phi$ SG005 genome at CDS11.

\subsection{Prophage Elements in S. gordonii ATCC10558}

To exclude the possibility that $\Phi S G 005$ was derived from the host $S$. gordonii genome, we detected prophage elements in the S. gordonii ATCC10558 chromosome, which is composed of 2,187,611 bp with a G+C content of $49.52 \%$ and 2039 CDSs (Table 1). Although PHASTER analysis predicted three prophage-related elements in the host genome (Regions 1-3, Figure S1), every region revealed incomplete prophage sequences. Each region was composed of hypothetical proteins (HYP) and phage-like protein (PLP), as shown in Figure S1B-D and Table S1. Only Region 1 exhibited the presence of attachment sites, attL and $a t t R$. These sequences showed no similarity with that of $\Phi S G 005$ in terms of the length, predicted CDSs functions, or genomic organization (Figures $3 \mathrm{~A}$ and $\mathrm{S1}$ ).

\subsection{Molecular Characterization of $\Phi S G 005$}

Phylogenetic analysis was conducted to clarify the relationships between known streptococcus viruses and $\Phi S G 005$. As shown in Figure 4, a phylogenetic tree constructed using whole-genome sequences by VICTOR yielded fifteen species, four genus, and two family clusters. $\Phi S G 005$ forms a distinct clade among the streptococcus viruses, but shares common evolutionary origins with streptococcus virus C1, indicating that $\Phi S G 005$ is a unique species and independent from any other known streptococcus virus. The intergenomic similarity between streptococcus virus C1 and $\Phi S G 005$ was $13.11 \%$. Further 
phylogenetic analysis was performed using DNA polymerase (CDS15), tail fiber (CDS11), endolysin (CDS13), and CHAP-domain containing lysin (CDS12) sequences to determine the molecular characteristics of $\Phi S G 005$. The phylogenetic tree constructed using the DNA polymerase sequence revealed that it formed an independent branch located close to streptococcus virus C1 DNA polymerase (Figure 5A). In addition, phylogenetic analysis of the tail fiber demonstrated that the $\Phi S G 005$ tail fiber is most closely related to streptococcus virus $\mathrm{C} 1$ tail proteins (Figure 5B). A phylogenetic tree constructed using endolysin resulted in a distinct clade among the streptococcal endolysins, but CHAP-domain containing lysin formed an independent branch and shared ancestral roots with phage lysin derived from streptococcus virus C1 (PlyC, Figure 5C,D). These data indicated that $\Phi S G 005$ shares the most recent common ancestor with streptococcus virus $\mathrm{C} 1$, but forms a distinct clade among the streptococcus viruses.

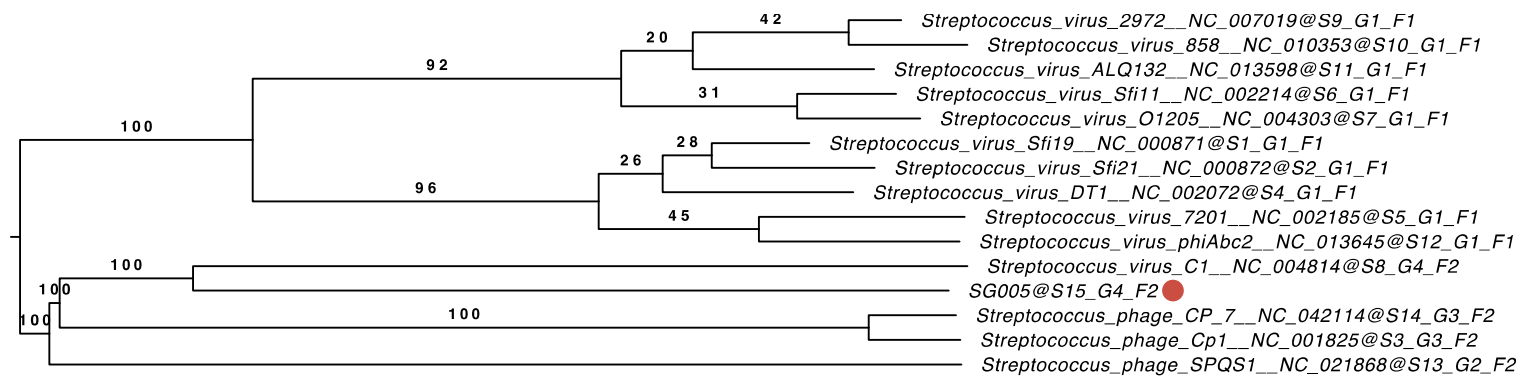

0.06

Figure 4. Phylogenetic analysis using the whole-genome sequence of $\Phi$ SG005. The phylogenetic tree was constructed by VICTOR using 14 whole-genome sequences of all streptococcus master species registered by the International Committee on the Taxonomy of Viruses (ICTV). The red circle indicates $\Phi S G 005 . ~ S, ~ G$, and F after the phage names refer to species, genus, and family clusters, respectively.
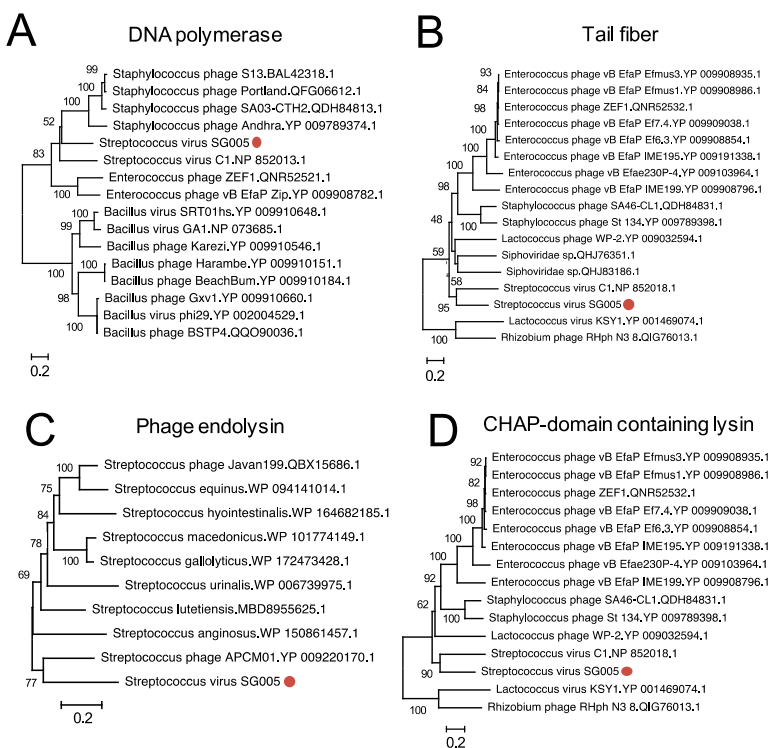

Figure 5. Phylogenetic analysis of some CDSs of $\Phi S G 005$. Phylogenetic trees of DNA polymerase (A), tail fiber protein (B), endolysin (C), and CHAP-domain containing lysin (D) were constructed by the neighbor-joining method (1000 replications) based on the full-length amino acid sequences. Bootstrap values are shown on the branch nodes, and the scale bar represents a distance of 0.2 substitutions per site. Red circles indicate $\Phi S G 005$. 


\section{Discussion}

In the present study, we discovered a novel virulent streptococcus virus, $\Phi S G 005$. Molecular characterization of the virus revealed that $\Phi S G 005$ possesses an independent and unique sequence compared to previously known phages, so $\Phi S G 005$ expands our understanding of the molecular features of Streptococcus viruses. $\Phi$ SG005 was found to be related to streptococcus virus $\mathrm{C} 1$, which was isolated from a sewage plant in Milwaukee in 1925 as a virulent phage [30]. It has taken nearly 100 years to discover a virus that forms a distinct clade next to streptococcus virus $\mathrm{C} 1$; however, our findings make us optimistic that undiscovered streptococcus viruses harboring unique molecular characteristics still exist in nature. In addition, spacer targeting analysis has received attention as a novel prediction tool to search for phages [31,32], as CRISPR spacers are derived from previous infection by phage genomic elements; these sequences guarantee the presence of infectious phages towards host strains. Thus, our analysis indicated the presence of $\Phi$ SG005-related streptococcus viruses elsewhere, because the CRISPR tracer 1 sequence was not completely consistent with the $\Phi S G 005$ tail fiber sequence, which ensures the presence of diverse streptococcus viruses.

The isolation of phages is affected by several factors. Notably, multiple phageresistance systems are likely involved in the failure of phage isolations $[1,33]$. In the present study, we found that the host strain S. gordonii ATCC10558 contains a CRISPR spacer showing high sequence similarity with the middle region of the QSG005 tail fiber sequence, suggesting that $S$. gordonii ATCC 10558 has previously been infected with phages similar to $\Phi S G 005$. A previous study reported that S. pyogenes-derived Cas9 tolerates nucleotide mismatches between the guide RNA and target DNA. Notably, multiple protospacer adjacent motif (PAM)-distal mismatches can be tolerated for Cas9 nucleotide digestion [13,34]. As shown in Figure 3B, a mismatched 7-nt between CRISPR spacer 1 and the $\Phi S G 005$ target DNA position in the PAM-distal region (5' of CRISPR RNA: crRNA) suggests that this crRNA might recognize the genome of $\Phi S G 005$ during infection, leading to phage-resistance; however, our bioinformatics analysis estimated that $\Phi S G 005$ encodes an AcrIIA5-like protein, which functions as type II Cas9 inhibitor as a counter-defense mechanism [35]. As S. gordonii ATCC10558 harbors a type II CRISPR/Cas system, these data suggested that we could isolate novel virulent phages fortuitously due to the $\Phi$ SG005encoded anti-CRISPR system, because the bacterial adaptive immune response is possibly neutralized by a phage-encoded AcrIIA5-like protein. In order to isolate novel undiscovered lytic phages such as S. agalactiae phages, we have to develop further sophisticated isolation methods avoiding phage-resistance mechanisms as represented by CRISPR/Cas9. To this end, further understanding of the counter-defense systems encoded by phages is required.

The bactericidal effects of phages can be applied as treatments for bacterial infections, so-called phage therapy. In these therapies, virulent phages should be applied to achieve desirable outcomes, because temperate phages may transfer toxic or pathogenic genes [2,36]. Thus, whether we can implement phage therapy efficiently depends on the availability of virulent phages. In fact, phage therapy against S. agalactiae and Staphylococcus pseudintermedius infection has still not been developed well, because virulent phages with efficient bactericidal effects against these bacteria have not been found [10-12]. In this context, the first isolated virulent phage against $S$. gordonii, $\Phi S G 005$, that possesses a bactericidal effect (Figure 2) has the potential to be developed into a phage therapy against $S$. gordonii infection. Notably, phages exhibit specific infectivity against bacteria, which allows for phage therapy without the disruption of associated normal microbiota. Therefore, further characterization of the $\Phi S G 005$ lytic capability against diverse oral bacteria, including different S. gordonii isolates, would expand the potential of this virulent phage for therapy. In addition, some kinds of phage-derived recombinant proteins, such as endolysins and tail-associated enzymes, by themselves eliminate or reduce bacteria $[37,38]$, suggesting a unique antimicrobial strategy. Notably, despite streptococcus virus $\mathrm{C} 1$ infecting only group C streptococcus [39,40], the endolysin PlyC derived from streptococcus virus C1, 
which is closely related to CHAP-domain containing lysin (CDS13, Figure 5D), has potent lytic activity against group A, C, and E streptococcus strains [41]. Thus, $\Phi$ SG005-encoded lysins may contribute to the development of alternative approaches against $S$. gordonii.

In the present study, we isolated for the first time a virulent streptococcus virus, $\Phi S G 005$, that infects S. gordonii. These findings facilitate our understanding of streptococcus viruses and have potential for developing phage therapy against $S$. gordonii infection.

Supplementary Materials: The following are available online at https:/ /www.mdpi.com/article/10.3 390/v13101964/s1, Figure S1: Identification of prophage-associated regions in S. gordonii ATCC10558, Table S1: Products and predicted functions of proteins encoded by each region described in Figure S1B-D.

Author Contributions: Conceptualization, J.F. and H.I.; Data curation, K.N. (Keisuke Nakamura), Y.A. and K.N. (Keita Nishida); Formal analysis, T.N., K.N. (Keisuke Nakamura), Y.A. and K.N. (Keita Nishida); Funding acquisition, J.F., H.K., H.H. and H.I.; Investigation, J.F., S.-i.Y., T.N., K.N (Keisuke Nakamura), Y.A., K.N. (Keita Nishida), M.S., T.I. and H.S.; Project administration, H.K. and H.H.; Resources, K.N. (Keitaro Nishi), H.K. and H.H.; Software, T.N.; Supervision, H.I.; Validation, H.I.; Visualization, J.F.; Writing—original draft, J.F.; Writing—review \& editing, H.I. All authors have read and agreed to the published version of the manuscript.

Funding: This study was supported by grants for Scientific Research on Innovative Areas and International Group from the Ministry of Education, Culture, Sports, Science, and Technology, Japan (MEXT)/JSPS KAKENHI (JP20K09923, JP20K21680, JP19K15985 and JP17H01506), and a grant from MEXT for the Joint Research Program of the Hokkaido University International Institute for Zoonosis Control.

Institutional Review Board Statement: Not applicable.

Informed Consent Statement: Not applicable.

Data Availability Statement: In this section, please provide details regarding where data supporting reported results can be found, including links to publicly archived datasets analyzed or generated during the study. Please refer to suggested Data Availability Statements in section "MDPI Research Data Policies" at https:/ /www.mdpi.com/ethics (accessed on 10 September 2021). You might choose to exclude this statement if the study did not report any data.

Conflicts of Interest: The authors declare that the research was conducted in the absence of any commercial or financial relationships. The author SY is an employee of KANEKA \& Co., Ltd., Japan.

\section{References}

1. Hampton, H.G.; Watson, B.N.J.; Fineran, P.C. The arms race between bacteria and their phage foes. Nature 2020, 577, 327-336. [CrossRef] [PubMed]

2. Kortright, K.E.; Chan, B.K.; Koff, J.L.; Turner, P.E. Phage therapy: A renewed approach to combat antibiotic-resistant bacteria. Cell. Host Microbe 2019, 25, 219-232. [CrossRef]

3. Fan, H.F.; Su, B.Y.; Ma, C.H.; Rowley, P.A.; Jayaram, M. A bipartite thermodynamic-kinetic contribution by an activating mutation to rdf-independent excision by a phage serine integrase. Nucleic Acids Res. 2020, 48, 6413-6430. [CrossRef] [PubMed]

4. Synnott, A.J.; Kuang, Y.; Kurimoto, M.; Yamamichi, K.; Iwano, H.; Tanji, Y. Isolation from sewage influent and characterization of novel staphylococcus aureus bacteriophages with wide host ranges and potent lytic capabilities. Appl. Env. Microbiol. 2009, 75, 4483-4490. [CrossRef] [PubMed]

5. Furusawa, T.; Iwano, H.; Hiyashimizu, Y.; Matsubara, K.; Higuchi, H.; Nagahata, H.; Niwa, H.; Katayama, Y.; Kinoshita, Y.; Hagiwara, K.; et al. Phage therapy is effective in a mouse model of bacterial equine keratitis. Appl. Env. Microbiol. 2016, 82, 5332-5339. [CrossRef] [PubMed]

6. Fujiki, J.; Furusawa, T.; Munby, M.; Kawaguchi, C.; Matsuda, Y.; Shiokura, Y.; Nakamura, K.; Nakamura, T.; Sasaki, M.; Usui, M.; et al. Susceptibility of pseudomonas aeruginosa veterinary isolates to pbunavirus pb1-like phages. Microbiol. Immunol. 2020, 64, 778-782. [CrossRef] [PubMed]

7. Duan, Y.; Llorente, C.; Lang, S.; Brandl, K.; Chu, H.; Jiang, L.; White, R.C.; Clarke, T.H.; Nguyen, K.; Torralba, M.; et al. Bacteriophage targeting of gut bacterium attenuates alcoholic liver disease. Nature 2019, 575, 505-511. [CrossRef]

8. Veyrand-Quiros, B.; Guzman-Villanueva, L.T.; Reyes, A.G.; Rodriguez-Jaramillo, C.; Salas-Leiva, J.S.; Tovar-Ramirez, D.; Balcazar, J.L.; Quiroz-Guzman, E. Assessment of bacteriophage vb_pd_pdcc-1 on bacterial dynamics during ontogenetic development of the longfin yellowtail (seriola rivoliana). Appl. Microbiol. Biotechnol. 2021, 105, 2877-2887. [CrossRef] 
9. Shimamori, Y.; Mitsunaka, S.; Yamashita, H.; Suzuki, T.; Kitao, T.; Kubori, T.; Nagai, H.; Takeda, S.; Ando, H. Staphylococcal phage in combination with staphylococcus epidermidis as a potential treatment for staphylococcus aureus-associated atopic dermatitis and suppressor of phage-resistant mutants. Viruses 2020, 13, 7. [CrossRef]

10. Furfaro, L.L.; Chang, B.J.; Payne, M.S. Applications for bacteriophage therapy during pregnancy and the perinatal period. Front. Microbiol. 2017, 8, 2660. [CrossRef]

11. Moodley, A.; Kot, W.; Nalgard, S.; Jakociune, D.; Neve, H.; Hansen, L.H.; Guardabassi, L.; Vogensen, F.K. Isolation and characterization of bacteriophages active against methicillin-resistant staphylococcus pseudintermedius. Res. Vet. Sci. 2019, 122, 81-85. [CrossRef] [PubMed]

12. Kim, S.G.; Giri, S.S.; Yun, S.; Kim, S.W.; Han, S.J.; Kwon, J.; Oh, W.T.; Lee, S.B.; Park, Y.H.; Park, S.C. Two novel bacteriophages control multidrug- and methicillin-resistant staphylococcus pseudintermedius biofilm. Front. Med. (Lausanne) 2021, 8, 524059. [CrossRef]

13. Jinek, M.; Chylinski, K.; Fonfara, I.; Hauer, M.; Doudna, J.A.; Charpentier, E. A programmable dual-rna-guided DNA endonuclease in adaptive bacterial immunity. Science 2012, 337, 816-821. [CrossRef] [PubMed]

14. Charpentier, E.; Richter, H.; van der Oost, J.; White, M.F. Biogenesis pathways of rna guides in archaeal and bacterial crispr-cas adaptive immunity. FEMS Microbiol. Rev. 2015, 39, 428-441. [CrossRef] [PubMed]

15. van der Ploeg, J.R. Characterization of streptococcus gordonii prophage ph15: Complete genome sequence and functional analysis of phage-encoded integrase and endolysin. Microbiology 2008, 154, 2970-2978. [CrossRef] [PubMed]

16. Guo, G.; Du, D.; Yu, Y.; Zhang, Y.; Qian, Y.; Zhang, W. Pan-genome analysis of streptococcus suis serotype 2 revealed genomic diversity among strains of different virulence. Transbound Emerg. Dis. 2020, 14, 156. [CrossRef]

17. Rezaei Javan, R.; Ramos-Sevillano, E.; Akter, A.; Brown, J.; Brueggemann, A.B. Prophages and satellite prophages are widespread in streptococcus and may play a role in pneumococcal pathogenesis. Nat. Commun. 2019, 10, 4852. [CrossRef] [PubMed]

18. Park, O.J.; Kwon, Y.; Park, C.; So, Y.J.; Park, T.H.; Jeong, S.; Im, J.; Yun, C.H.; Han, S.H. Streptococcus gordonii: Pathogenesis and host response to its cell wall components. Microorganisms 2020, 8, 1852. [CrossRef]

19. Back, C.R.; Sztukowska, M.N.; Till, M.; Lamont, R.J.; Jenkinson, H.F.; Nobbs, A.H.; Race, P.R. The streptococcus gordonii adhesin csha protein binds host fibronectin via a catch-clamp mechanism. J. Biol. Chem. 2017, 292, 1538-1549. [CrossRef]

20. Loo, C.Y.; Corliss, D.A.; Ganeshkumar, N. Streptococcus gordonii biofilm formation: Identification of genes that code for biofilm phenotypes. J. Bacteriol. 2000, 182, 1374-1382. [CrossRef]

21. Mosailova, N.; Truong, J.; Dietrich, T.; Ashurst, J. Streptococcus gordonii: A rare cause of infective endocarditis. Case Rep. Infect. Dis. 2019, 2019, 7127848. [CrossRef]

22. Cheng, J.; Hu, H.; Fang, W.; Shi, D.; Liang, C.; Sun, Y.; Gao, G.; Wang, H.; Zhang, Q.; Wang, L.; et al. Detection of pathogens from resected heart valves of patients with infective endocarditis by next-generation sequencing. Int. J. Infect. Dis. 2019, 83, 148-153. [CrossRef]

23. Washburn, M.R.; White, J.C.; Niven, C.F.; Streptococcus, B.E. Immunological characteristics. J. Bacteriol. 1946, 51, 723-729. [CrossRef]

24. Bonilla, N.; Rojas, M.I.; Netto Flores Cruz, G.; Hung, S.H.; Rohwer, F.; Barr, J.J. Phage on tap-a quick and efficient protocol for the preparation of bacteriophage laboratory stocks. PeerJ 2016, 4, e2261. [CrossRef]

25. Khan Mirzaei, M.; Nilsson, A.S. Isolation of phages for phage therapy: A comparison of spot tests and efficiency of plating analyses for determination of host range and efficacy. PLoS ONE 2015, 10, e0118557. [CrossRef]

26. Meier-Kolthoff, J.P.; Goker, M. Victor: Genome-based phylogeny and classification of prokaryotic viruses. Bioinformatics 2017, 33, 3396-3404. [CrossRef]

27. Kumar, S.; Stecher, G.; Li, M.; Knyaz, C.; Tamura, K. Mega x: Molecular evolutionary genetics analysis across computing platforms. Mol. Biol. Evol. 2018, 35, 1547-1549. [CrossRef]

28. Moraru, C.; Varsani, A.; Kropinski, A.M. Viridic-a novel tool to calculate the intergenomic similarities of prokaryote-infecting viruses. Viruses 2020, 12, 1268. [CrossRef]

29. Ackermann, H.W. 5500 phages examined in the electron microscope. Arch. Virol. 2007, 152, 227-243. [CrossRef]

30. Clark, P.F.; Clark, A.S. A “bacteriophage” active against a hemolytic streptococcus. J. Bacteriol. 1926, 11, 142. [CrossRef]

31. Biswas, A.; Gagnon, J.N.; Brouns, S.J.; Fineran, P.C.; Brown, C.M. Crisprtarget: Bioinformatic prediction and analysis of crrna targets. RNA Biol. 2013, 10, 817-827. [CrossRef]

32. Boudry, P.; Semenova, E.; Monot, M.; Datsenko, K.A.; Lopatina, A.; Sekulovic, O.; Ospina-Bedoya, M.; Fortier, L.C.; Severinov, K.; Dupuy, B.; et al. Function of the crispr-cas system of the human pathogen clostridium difficile. MBio 2015, 6, e01112-e01115. [CrossRef]

33. Rostol, J.T.; Marraffini, L. (ph)ighting phages: How bacteria resist their parasites. Cell. Host Microbe 2019, 25, 184-194. [CrossRef]

34. Jiang, W.; Bikard, D.; Cox, D.; Zhang, F.; Marraffini, L.A. Rna-guided editing of bacterial genomes using crispr-cas systems. Nat. Biotechnol. 2013, 31, 233-239. [CrossRef]

35. An, S.Y.; Ka, D.; Kim, I.; Kim, E.H.; Kim, N.K.; Bae, E.; Suh, J.Y. Intrinsic disorder is essential for cas9 inhibition of anti-crispr acriia5. Nucleic Acids Res. 2020, 48, 7584-7594. [CrossRef]

36. Fortier, L.C.; Sekulovic, O. Importance of prophages to evolution and virulence of bacterial pathogens. Virulence 2013, 4, 354-365. [CrossRef] 
37. Paul, V.D.; Rajagopalan, S.S.; Sundarrajan, S.; George, S.E.; Asrani, J.Y.; Pillai, R.; Chikkamadaiah, R.; Durgaiah, M.; Sriram, B.; Padmanabhan, S. A novel bacteriophage tail-associated muralytic enzyme (tame) from phage $\mathrm{k}$ and its development into a potent antistaphylococcal protein. BMC Microbiol. 2011, 11, 226. [CrossRef]

38. Fujiki, J.; Nakamura, T.; Furusawa, T.; Ohno, H.; Takahashi, H.; Kitana, J.; Usui, M.; Higuchi, H.; Tanji, Y.; Tamura, Y.; et al Characterization of the lytic capability of a lysk-like endolysin, lys-phisa012, derived from a polyvalent staphylococcus aureus bacteriophage. Pharmacy 2018, 11, 456.

39. Lancefield, R.C. Note on the susceptibility of certain strains of hemolytic streptococcus to a streptococcus bacteriophage. Proc. Soc. Exp. Biol. Med. 1932, 30, 169-171. [CrossRef]

40. Shwartzman, G. Studies on streptococcus bacteriophage: I. A powerful lytic principle against hemolytic streptococci of erysipelas origin. J. Exp. Med. 1927, 46, 497-509. [CrossRef]

41. Nelson, D.; Loomis, L.; Fischetti, V.A. Prevention and elimination of upper respiratory colonization of mice by group a streptococci by using a bacteriophage lytic enzyme. Proc. Natl. Acad. Sci. USA 2001, 98, 4107-4112. [CrossRef] [PubMed] 